\title{
Eigenvalue Based Sensing and SNR Estimation for Cognitive Radio in Presence of Noise Correlation
}

\author{
Shree Krishna Sharma, Student Member, IEEE, Symeon Chatzinotas, Member, IEEE \\ and Björn Ottersten, Fellow, IEEE
}

\begin{abstract}
Herein, we present a detailed analysis of an eigenvalue based sensing technique in the presence of correlated noise in the context of a Cognitive Radio (CR). We use a Standard Condition Number (SCN) based decision statistic based on asymptotic Random Matrix Theory (RMT) for decision process. Firstly, the effect of noise correlation on eigenvalue based Spectrum Sensing (SS) is studied analytically under both the noise only and the signal plus noise hypotheses. Secondly, new bounds for the SCN are proposed for achieving improved sensing in correlated noise scenarios. Thirdly, the performance of Fractional Sampling (FS) based SS is studied and a method for determining the operating point for the FS rate in terms of sensing performance and complexity is suggested. Finally, a Signal to Noise Ratio (SNR) estimation technique based on the maximum eigenvalue of the received signal's covariance matrix is proposed. It is shown that proposed SCN-based threshold improves sensing performance in correlated noise scenarios and SNRs up to $0 \mathrm{~dB}$ can be reliably estimated with less than 1 $\%$ normalized Mean Square Error (MSE) in the presence of correlated noise without the knowledge of noise variance.
\end{abstract}

Index Terms-Spectrum Sensing, SNR Estimation, Noise Correlation, Random Matrix Theory.

\section{INTRODUCTION}

$\mathbf{S}$ PECTRUM Sensing (SS) plays an important role in Cognitive Radio (CR) networks in order to acquire the spectrum awareness required by CRs. The three main signal processing techniques for sensing the presence of a Primary User (PU) that appear in the literature are matched filter detection, Energy Detection (ED) and cyclostationary feature detection [1]. Matched filter detection and cyclostationary feature detection techniques require prior knowledge of the PU's signal to decide about the presence or absence of the PU signal [2]. Although ED technique does not require any prior knowledge of PU's signal, the performance of this technique is susceptible to noise covariance uncertainty [3]. Since both the prior knowledge about the PU's signal and the noise variance are unknown to the CRs in practical scenarios, exploring efficient and blind SS techniques for CRs has emerged as an important research challenge. Several blind SS techniques have been proposed in the literature [4-7] without requiring the prior knowledge of the PU's signal, the channel and the noise power. Furthermore, the performance of traditional SS

Copyright (c) 2013 IEEE. Personal use of this material is permitted. However, permission to use this material for any other purposes must be obtained from the IEEE by sending a request to pubs-permissions@ieee.org.

The authors are with the Interdisciplinary Centre for Security, Reliability and Trust (SnT) (http://www.securityandtrust.lu), University of Luxembourg, L-2721, Luxembourg. Email: \{shree.sharma, symeon.chatzinotas, bjorn.ottersten\}@uni.lu. techniques is limited by received signal strength which may be severely degraded in multi-path fading and shadowing environments. Different diversity enhancing techniques such as multi-antenna, cooperative and oversampled techniques have been introduced in the literature to enhance the SS efficiency in wireless fading channels [8-10]. Most of these methods use the properties of the eigenvalues of the received signal's covariance matrix and use recent results from advances in Random Matrix Theory (RMT) $[11,12]$. The main advantage of eigenvalue based SS over other SS techniques is that it does not require any prior information of the PU's signal and it outperforms ED techniques, especially in the presence of noise covariance uncertainty [8].

In this paper, we use the Standard Condition Number (SCN) of the noise covariance matrix to analyze the effect of noise correlation on eigenvalue based SS techniques. The $\mathrm{SCN}$ of a matrix is defined as the ratio of the maximum eigenvalue to the minimum eigenvalue [13] and can be used as a metric to characterize the support of the asymptotic eigenvalue probability distribution function (a.e.p.d.f.) of a random matrix. Furthermore, we use the SCN of the received signal's covariance matrix for decision process. If the calculated $\mathrm{SCN}$ is greater than noise only SCN, the decision is that a PU signal is present. Since noise correlation affects the SCN of the noise covariance matrix and as a result, the $\mathrm{SCN}$ of the received signal's covariance matrix, the decision metric is also affected. According to author's knowledge, this method has not been considered in the literature for SS in the presence of noise correlation.

Several blind SS techniques utilizing various features of the received signal's covariance matrix such as statistical covariance [14], autocorrelation [15] and eigenvalue distribution [8] have been proposed in the literature. In most of the existing eigenvalue based SS literature, the authors consider asymptotically large matrices whose eigenvalues are known to follow the Marchenko-Pastur (MP) law, which establishes the convergence of the largest and smallest eigenvalues of these matrices. The authors in [16] use this MP law to test a binary hypothesis under white noise conditions using the SCN for Wishart matrices. However, the sample covariance matrix of the noise is not a Wishart random matrix in the presence of correlated noise [8]. In practical situations, noise correlation arises due to oversampling and imperfections in filtering [8]. For example, when a received signal is filtered by a narrowband receive filter, the noise embedded in the received signal is also filtered and the output signal of the filter contains the correlated noise. In case of correlated noise scenarios, 
the eigenvalue distribution does not follow the MP law and the SCN threshold proposed in [16] may result in degraded PU sensing performance. Therefore, new SCN-based sensing thresholds need to be investigated for carrying out SS in the presence of noise correlation. This is the first contribution of this paper.

Furthermore, several methods based on eigenvalue distribution of received signal's covariance matrix usually focus on interweave CR meaning that a Secondary User (SU) transmits only when no PU signal is present $[8,9]$. However, if side information is available about the primary Signal to Noise Ratio (SNR), advanced underlay transmission schemes could be employed at the CRs. In practical scenarios, it would be advantageous to estimate the primary SNR in order to decide the transmission strategy of the cognitive transmitter. Depending on the estimated primary SNR level, different underlay transmission strategies (e.g., cognitive resource allocation) can be implemented at the cognitive transmitters to allow the coexistence of primary and secondary systems ${ }^{1}$. In this direction, we derive the a.e.p.d.f. of the received signal's covariance matrix for signal plus noise case under white and correlated noise scenarios. The a.e.p.d.f. is then used to determine the maximum eigenvalue which is in turn exploited to estimate the SNR. Moreover, the SNR estimation performance is evaluated based on normalized Mean Square Error (MSE). This is the second contribution of this paper.

The sampling rate in a CR receiver can be increased beyond the symbol rate, known as fractional sampling (FS), to enhance the SS efficiency under fading channel conditions. FS is commonly used to enhance signal detection reliability in the receiver [17-19]. From the CR point of view, an FS receiver can be modeled as a virtual multiple-output system with presumably independent channel fading effects. This technique is especially beneficial in time varying channels with large Doppler spread i.e., small channel coherence time. Another motivation for introducing the FS concept in the context of $\mathrm{CR}$ is that using more antennas at the receive-side is often impractical and expensive requiring multiple Radio Frequency (RF) chains. In wireless fading environments, FS introduces diversity and can improve signal detection. However, FS operation also results in colored noise [20] and this phenomenon gradually saturates the performance gain due to FS $[17,19]$. Therefore, it is important to determine the operating point for the FS rate, a design parameter that we can actually configure to find a good trade-off between performance and complexity. This is the third contribution of this paper.

The remainder of this paper is structured as follows: Section II reviews in detail prior work in the areas of eigenvalue based sensing. Section III describes the motivation for the considered problem and briefly summarizes the approach used in this paper. Section IV describes the considered signal models under white and correlated noise scenarios. Section V analyzes the effect of noise correlation for the noise only case and proposes new SCN-based decision bounds. Section VI provides the analysis for signal plus noise case under white and correlated

\footnotetext{
${ }^{1}$ In this context, we consider the scenario with short range primary and secondary wireless systems in which the interference levels from one system to another are at a similar level.
}

noise scenarios and describes the proposed eigenvalue based SNR estimation method. Section VII studies the performance of the proposed techniques with numerical simulations and proposes a method for determining the optimal FS operating point. Section VIII concludes the paper. The appendix includes some preliminaries on random matrix transforms.

\section{A. Notation}

Throughout the formulations of this paper, boldface upper and lower case letters are used to denote matrices and vectors respectively, $\mathbb{E}[\cdot]$ denotes expectation, $\mathbb{C}$ denotes the complex plane, $(\cdot)^{T}$ denotes the transpose matrix, $(\cdot)^{H}$ denotes the conjugate transpose matrix, $(\cdot)^{*}$ represents the complex conjugate, I denotes the identity matrix, $(z)^{+}$denotes $\max (0, z)$, $\mathbf{R}_{\mathbf{X}}$ represents the statistical covariance of $\mathbf{X}, \hat{\mathbf{R}}_{\mathbf{X}}$ represents the sample covariance of $\mathbf{X}, \mathcal{S}_{\mathbf{X}}$ represents Stieltjes transform of $\mathbf{X}, \mathcal{R}_{\mathbf{X}}$ represents $\mathrm{R}$ transform ${ }^{2}$ and $\Sigma_{\mathbf{X}}$ represents $\Sigma$ transform [11].

\section{RELATED WORK}

The three major eigenvalue based sensing techniques considered in the literature are [8]: Maximum-Minimum Eigenvalue (MME) detection, Energy with Minimum Eigenvalue (EME) detection and Maximum Eigenvalue Detection (MED). A number of eigenvalue based SS methods are proposed in $[8,10,16]$ utilizing eigenvalue properties of Wishart random matrices, which arise under noise only cases in white noise scenarios. The authors in [16] use MP law to test binary hypothesis problems. In [8], the Tracy-Widom (TW) distribution is used as a statistical model for the largest eigenvalue and both the TW distribution and the MP models are used to find the approximate distribution of random SCN. Subsequently, this distribution is used to derive the relationship between an expression for probability of false alarm $\left(P_{f}\right)$ and threshold. The difference between the MP approach and the TW approach is that MP is a deterministic function which characterizes the asymptotic matrix spectrum, while the TW approach provides the statistics of individual eigenvalues e.g., the maximum eigenvalue. Since the rate of convergence of the TW distribution is faster than MP law, the TW method is superior than the MP only method. However, the TW method outperforms the MP method only at relatively large SNRs since SCN is a ratio of two random variables and the approximation considered in [8] is accurate only for large SNR conditions.

In [10], an approximation of the threshold function is derived for systems having equal number of receiving antennas and samples. In [21], the p.d.f. of the eigenvalue ratio has been derived using the expression of the joint distributions of an arbitrary subset of ordered eigenvalues of complex Wishart matrices. In this scenario, the receiver should be provided with a lookup table in order to calculate the proposed inverse $\mathrm{Cu}$ mulative Distribution Function (CDF) of the second-order TW distribution. The exact distribution of the condition number

\footnotetext{
${ }^{2}$ Readers should not confuse $\mathrm{R}$ transform notation $\mathcal{R}$ with the covariance matrix notation $\mathbf{R}$.
} 
of a complex Wishart matrix has been used to calculate the threshold expression in [10] without the need of a lookup table. However, the calculated threshold expression in terms of $P_{f}$ in [10] is based on the exact density of the condition number of complex Wishart matrix considering the noise only case and it is only valid in case of white noise measurements. For the correlated noise scenarios, the sample covariance matrix does not follow the properties of Wishart random matrices.

In [22], a more accurate model considering the TracyWidom-Curtiss (TWC) model has been considered by using the distribution of the smallest eigenvalues of Wishart random matrices. However, the TW distribution and the Curtiss' ratio of variates formula are highly involved functions, which are hard to evaluate numerically and non tractable to find the support of a.e.p.d.f. [23]. In [23], the exact distribution of SCNs of dual Wishart random matrices has been used and it is argued that the proposed method requires only tens of samples and outperforms all the RMT based techniques. However, the authors in [23] considered the Wishart random matrix model for signal plus noise case for simplicity and did not address the fact that during the presence of signal and correlated noise, the sample covariance matrix may no longer be a Wishart random matrix. In [24], non-asymptotic behavior of eigenvalues of random matrices has been considered using the spectral properties of random sub-Gaussian matrices of fixed dimensions. A cooperative SS algorithm using double eigenvalue threshold has been proposed in [25], which considers two maximum eigenvalues for the noise only and the signal plus noise cases through analysis of sample covariance matrix of received signals using RMT approach. In [26], the effect of spatial correlation in the performance of predicted eigenvalue threshold based spectrum sensing is analyzed and it is shown that the detection performance improves in the presence of spatial correlation at the multi-antenna secondary user considering the white noise.

Spectrum sensing using free probability theory has also received important attention in the literature [27] [28]. In [27], a cooperative scheme for SS has been proposed using asymptotic free behavior of random matrices and the properties of Wishart distribution. The same work has been extended for MIMO scenario in [28]. In these works, the presence of the PU signal is decided simply by checking whether the power matrix is zero or not but this technique is not studied analytically in [27] and [28].

\section{PROBlem Description AND CONSIDERED APPROACH}

The eigenvalue based spectrum sensing techniques using RMT have been well investigated in various literature $[9,10$, 16, 22-24, 27]. However, most of these contributions assume the presence of white noise at the CR terminal. In practical implementation of a $\mathrm{CR}$, the received signal should pass through a pulse shaping filter before further processing. As an example, a typical block diagram of a wideband Software Defined Radio (SDR) receiver has been shown in Fig. 1 [29]. It mainly consists of three parts: wideband RF frontend, digital back-end and control part with processor. The noise

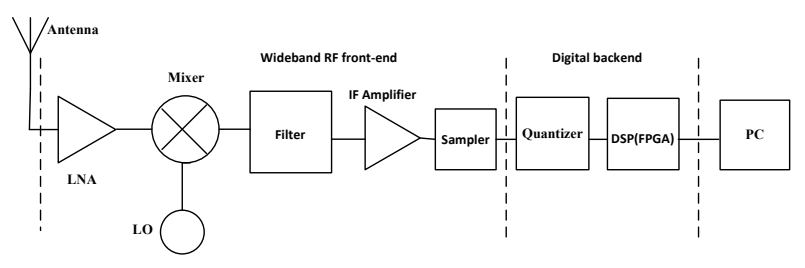

Fig. 1: Block diagram of a wideband SDR receiver

correlation at the CR sensing terminal may arise mainly due to the following two reasons [8]:

1) Filtering: The white noise is converted to the colored noise when passing through a dynamic system, typically a low pass filter, also called the shaping filter. When a received signal is passed through a shaping filter at the input of the receiver, the noise added to the signal before filtering also passes through the same filter. In this case, the noise covariance matrix depends on the transfer function of the pulse shaping filter used at the RF front end of a CR. The output signal of the shaping filter contains the colored noise and the color of the colored noise can be tuned by adjusting the parameters of the shaping filter.

2) Oversampling: When the shaping filter has a bandwidth of $B$ (which is usually equal to the bandwidth of the signal) but we sample at a rate higher than $2 B$, then the noise process in the (sampled) output is correlated even if the input noise process is white.

Due to absence of knowledge about the channel and the PU signal, coherent receivers such as matched filter i.e., receive part of root raised cosine filter) are not suitable for the SS applications. Active RC filters with tunable cut off frequencies has been proposed in the literature for CR applications [30, 31]. When a white noise input process with power spectral density $N_{0} / 2$ is the input to a RC filter with time constant $\mathrm{RC}$, the noise is colored after filtering. Although the channel may also get correlated at the output of the filter, we are interested in analyzing the effect of noise correlation on SS performance in this work assuming that noise correlation effect dominates the overall effect. The RC filter transforms the input autocorrelation function of white noise into output autocorrelation function given by [32]: $R_{y}(\nu)=\frac{N_{0}}{4 R C} e^{-\frac{|\nu|}{R C}}$. Since the autocorrelation function of output process of RC filter resembles the exponential model, we consider an exponential correlation model (see Section IV A) in this work. Since the same signal after being amplified through an Intermediate Frequency (IF) filter passes to the Analog to Digital Converter (ADC) (with reference to block diagram shown in Fig. 1), the correlation which may occur due to oversampling operation at the receiver gives rise to a similar correlation function as in case of the shaping filter.

In the context of eigenvalue based blind SS, MME and EME techniques have been proposed in the literature $[8,16$, 22]. The EME technique decides the presence or absence of the PUs by comparing the ratio of average eigenvalue $\left(\lambda_{\text {avg }}\right)$ to the minimum eigenvalue $\left(\lambda_{\min }\right)$ i.e., $\frac{\lambda_{\text {avg }}}{\lambda_{\text {min }}}$ with a predetermined threshold which is calculated based on the value 
of $P_{f}$. Similarly, the MME technique decides the presence or absence of primary users by comparing the ratio of the maximum eigenvalue $\left(\lambda_{\max }\right)$ to the minimum i.e., $\frac{\lambda_{\max }}{\lambda_{\min }}$ with the predetermined threshold. In [8], it is shown that MME technique performs better in comparison to the EME technique in terms of sensing performance. Regarding MME technique, different approaches such as asymptotic [16], semi-asymptotic [8] and ratio-based [22] approaches have been proposed in the literature assuming the presence of white noise.

In this paper, we consider two fundamental aspects of a CR, i.e., spectrum sensing and SNR estimation problems in the presence of noise correlation. We study and analyze both the problems with the same methodology. To analyze the sensing performance in the presence of noise correlation, we consider probability of correct decision ${ }^{3}$ as a performance metric by taking account of correct decisions under both hypotheses. The reason for choosing this metric is that the eigenvalue distribution of noise in the presence of correlation changes with respect to its eigenvalue distribution in the absence of correlation and we need to account for correct decisions under both hypotheses. Figure $2^{4}$ shows the performance of MME and EME techniques proposed in [8] and MP based asymptotic approach proposed in [16] in the presence of noise correlation. From the figure, it can be noted that noise correlation degrades the sensing performance of the considered detectors. In this context, exploring new sensing techniques which can provide better sensing efficiency in the presence of noise correlation is an important research challenge [33]. Motivated by this aspect, we propose new SCN-based decision bounds to improve the sensing performance in the presence of noise correlation with the help of theoretical analysis and numerical evaluation under noise only hypothesis (see Section V).

For modeling the noise correlation, we consider one-sided correlation model and then we use an exponential correlation model to define the components of the correlation matrix (see Section IV A). Moreover, for our considered FS sensing example, we use a simple linear model to vary the level of correlation with the FS rate (see Section V A). For carrying out theoretical analysis, we use an asymptotic approach as carried out in several literature [16, 27, 28, 34]. In this context, we use two important theorems ${ }^{5}$ in Section $\mathrm{V}$ from the RMT literature. These theorems are applied to find the a.e.p.d.f. of the received signal's covariance matrix under noise only hypothesis for white noise and correlated noise scenarios. The crossing points of these a.e.p.d.f.s with the $\mathrm{x}$-axis provide the decision bounds under considered scenarios.

Furthermore, the SNR estimation techniques in the white noise scenario may not perform well in the presence of noise correlation. Motivated by this aspect, we carry out detailed theoretical analysis under signal plus noise hypothesis to obtain the a.e.p.d.f. of the received signal's covariance matrix for white and correlated noise scenarios (see Section VI). Based on this a.e.p.d.f. of the received signal's covariance

\footnotetext{
${ }^{3}$ The definition of this metric is provided in Section VII.

${ }^{4}$ The simulation parameters used for getting this result are presented in Section VII.

${ }^{5}$ The details on these theorems can be found in [11] and [35] and we do not include their proofs in this paper due to space limitation.
}

matrix, we propose an SNR estimation method for estimating the SNR of the PU signal using the maximum eigenvalue (see Section VI C).

\section{Signal Model}

Let us consider a single cognitive user and a single primary user for simplicity of analysis. Let $N$ be the number of samples analyzed by the cognitive user for the decision process in the time duration of $\tau$ while performing symbol rate sampling. The sampling rate in the receiver can be increased beyond the symbol rate to enhance the signal detection capability in wireless fading channels. This technique known as FS [17] produces $N$ FS samples out of each original sample. Let $M$ be the FS rate carried out at the input of cognitive receiver. From signal model point of view, this factor can be considered as the number of multiple outputs analogous to the number of cooperating users in cooperative based sensing or the number of antennas in multiple antenna sensing as considered in related literature $[8,16]$. We denote the hypotheses of the presence and absence of the PU signal by $\mathbb{H}_{1}$ and $\mathbb{H}_{0}$ respectively. A binary hypothesis testing problem for $k$-th FS branch, $k=1, \ldots, M$, can be written as:

$$
\begin{aligned}
& \mathbb{H}_{0}: y_{k}(i)=\hat{z}_{k}(i) \\
& \text { PU absent } \\
& \mathbb{H}_{1}: y_{k}(i)=h_{k}(i) s(i)+\hat{z}_{k}(i) \text {, } \\
& \text { PU present }
\end{aligned}
$$

where $y_{k}(i)$ is the signal observed by the $k$-th receiving dimension at the $i$-th instant, $i=1,2, . ., N, s(i)$ is the PU signal at the $i$-th instant, which is to be detected, $h_{k}(i)$ is the amplitude gain of the channel for the $k$-th receive dimension at the $i$-th instant, and $\hat{z}_{k}(i)$ denotes the colored noise for the $k$-th receive dimension at the $i$-th instant. For our analysis, we assume that transmitted symbols are independent and identically distributed (i.i.d.) complex circularly symmetric (c.c.s.) Gaussian symbols, the noise samples in each FS branch are independent but are correlated across FS branches.

The $M \times N$ channel matrix $\mathbf{H}$ consists of i.i.d. coefficients and each row of $\mathbf{H}$ represents the channel coefficients for $N$ number of samples for each FS branch i.e., $\mathbf{H} \triangleq\left[\mathbf{h}_{1}^{T}, \mathbf{h}_{2}^{T}, \ldots, \mathbf{h}_{M}^{T}\right]^{T}$, with $\mathbf{h}_{m} \triangleq$ $\left[\begin{array}{llll}h_{m}(1) & h_{m}(2) & \ldots & h_{m}(N)\end{array}\right]$ with $m=1,2, \ldots, M$. We assume channel coefficients to be i.i.d. in each FS branch and the channel coherence time to be sufficiently small so that channel is not correlated as we increase the FS rate.

While performing sensing in a cognitive receiver, the sensing duration $(\tau)$ and symbol interval $\left(T_{s}\right)$ may not be the same depending on the signal bandwidth and sampling rate used at the receiver. For example, let us consider a coexistence scenario of TV whitespace broadband and wireless microphone systems. These are two systems with different operation bandwidths, a microphone signal typically occupies $200 \mathrm{kHz}$ bandwidth while TV signal occupies $6 \mathrm{MHz}$ and microphone operates on TV bands [8]. In this scenario, $\tau$ becomes much greater than $T_{s}$. Under the $\mathbb{H}_{1}$ hypothesis, we consider the following signal models considering the relation between $\tau$ and $T_{s}$.

Case 1: In this case, we consider that the transmitted symbol remains constant during the sensing period. This case may 
result when the sampling rate at the receiver is much higher than the transmitted symbol rate. The $M \times N$ received signal matrix $\mathbf{Y}$ in this case can be written as: $\mathbf{Y}=\sqrt{p} \mathbf{H} s+\hat{\mathbf{Z}}$, where $s$ is a constant transmitted symbol, $p$ is the power of transmitted symbol and $\hat{\mathbf{Z}} \triangleq\left[\hat{\mathbf{z}}_{1}^{T}, \hat{\mathbf{z}}_{2}^{T}, \ldots, \hat{\mathbf{z}}_{M}^{T}\right]^{T}$, with $\hat{\mathbf{z}}_{m} \triangleq\left[\begin{array}{llll}\hat{z}_{m}(1) & \hat{z}_{m}(2) & \ldots & \hat{z}_{m}(N)\end{array}\right]$. Since we assume the normalized unit value of noise variance, $\mathrm{SNR} \equiv p$. In this case, the sample covariance of transmitted signal can be written as: $R_{s}=\mathbb{E}\left[s^{2}\right]=1$.

Case 2: In this case, each symbol rate sampling period i.e., each column of matrix $\mathbf{Y}$, includes the samples for a single symbol. $\mathbf{Y}=\sqrt{p} \mathbf{H} \mathbf{S}_{d}+\hat{\mathbf{Z}}$, where $\mathbf{S}_{d}$ is the diagonal transmitted signal matrix of dimension $N \times N$ with diagonal $\mathbf{s}=[s(1) \ldots s(N)]$. In this case, the sample covariance matrix of the transmitted signal becomes

$$
\mathbf{R}_{\mathbf{S}}=\mathbb{E}\left[\mathbf{S}_{d} \mathbf{S}_{d}^{H}\right]=\left[\begin{array}{cccc}
\mathbb{E}\left[s^{2}(1)\right] & 0 & \cdots & 0 \\
0 & \mathbb{E}\left[s^{2}(2)\right] & \cdots & 0 \\
& & \ddots & \\
0 & 0 & \cdots & \mathbb{E}\left[s^{2}(N)\right]
\end{array}\right]=\mathbf{I}
$$

assuming that for each sample we get an i.i.d. c.c.s. Gaussian symbol with $\mathbb{E}\left[s^{2}\right]=1$.

The received signal matrix $\mathbf{Y}$ in both cases can be written in the following form:

$$
\mathbf{Y}=\left[\begin{array}{c}
\mathbf{y}_{1} \\
\mathbf{y}_{2} \\
\vdots \\
\mathbf{y}_{M}
\end{array}\right]=\left[\begin{array}{cccc}
y_{1}(1) & y_{1}(2) & \ldots & y_{1}(N) \\
y_{2}(1) & y_{2}(2) & \ldots & y_{2}(N) \\
\vdots & \vdots & \ddots & \vdots \\
y_{M}(1) & y_{M}(2) & \ldots & y_{M}(N)
\end{array}\right]
$$

Assuming that the source signal is independent from the noise, the covariance matrix of received signal, $\mathbf{R}_{\mathbf{Y}}$, is given by [8];

$$
\begin{aligned}
\mathbf{R}_{\mathbf{Y}} & =\mathbb{E}\left[\mathbf{Y} \mathbf{Y}^{H}\right]=\mathbb{E}\left[(\sqrt{p} \mathbf{H S})(\sqrt{p} \mathbf{H S})^{H}\right]+\mathbb{E}\left[\hat{\mathbf{Z}} \hat{\mathbf{Z}}^{H}\right] \\
& =p \mathbb{E}\left[\mathbf{H} \mathbf{H}^{H}\right]+\mathbf{R}_{\hat{\mathbf{Z}}}
\end{aligned}
$$

where $\mathbf{R}_{\hat{\mathbf{Z}}}=\mathbb{E}\left[\hat{\mathbf{Z}} \hat{\mathbf{Z}}^{H}\right]$. Let us define sample covariance matrices of received signal and noise as: $\hat{\mathbf{R}}_{\mathbf{Y}}(N)=\frac{1}{N} \mathbf{Y} \mathbf{Y}^{H}$ and $\hat{\mathbf{R}}_{\hat{\mathbf{Z}}}(N)=\frac{1}{N} \hat{\mathbf{Z}} \hat{\mathbf{Z}}^{H}$. The received signal $\mathbf{Y}$ can be further written as:

$$
\mathbf{Y}= \begin{cases}\sqrt{p} \mathbf{H} s+\hat{\mathbf{Z}}, & \text { Case } 1 \\ \sqrt{p} \mathbf{H S}_{d}+\hat{\mathbf{Z}}, & \text { Case } 2\end{cases}
$$

where $\hat{\mathbf{Z}} \sim \mathcal{C N}\left(0, \hat{\mathbf{R}}_{\hat{\mathbf{Z}}}(N)\right)$ is the colored noise. The $\mathrm{SCN}$ of $\hat{\mathbf{R}}_{\hat{\mathbf{Z}}}(N)$ depends on the noise correlation among noise samples across FS branches.

\section{A. Noise Correlation Modeling}

To analyze the noise correlation effect mathematically, a simple correlation model should be employed. In this work, we consider one-sided noise correlation model. With this model, the colored noise can be related to the white noise using the following expression.

$$
\hat{\mathbf{Z}}=\Theta^{1 / 2} \mathbf{Z}
$$

where $\mathbf{Z}$ is an $M \times N$ matrix with c.c.s. i.i.d. Gaussian entries with zero mean and unit variance, representing the white noise and $\Theta$ is an $M \times M$ Hermitian matrix whose entries correspond to the correlation among noise samples across FS branches and $\Theta^{1 / 2}$ denotes the square root of $\Theta$. To ensure that $\Theta$ does not affect the noise power, we consider the following normalization:

$$
(1 / M) \operatorname{trace}\{\boldsymbol{\Theta}\}=1 .
$$

The exponential correlation model can be written as $[36,37]$ :

$$
\theta_{i j}= \begin{cases}\rho^{(j-i)}, & i \leq j \\ \left(\rho^{(i-j)}\right)^{*}, & i>j\end{cases}
$$

where $\theta_{i j}$ is the $(i, j)$-th element of $\boldsymbol{\Theta}$ and $\rho \in \mathbb{C}$ is the correlation coefficient with $|\rho| \leq 1$.

\section{ANALYSIS UNDER $\mathbb{H}_{0}$ HYPOTHESIS}

RMT has been used in the literature in various applications such as modeling transmit/receive correlation in MIMO channels and multiuser MIMO fading [35,37]. Here, we state two RMT based theorems which are going to be used in defining our decision statistics.

Theorem 5.1: [11] Consider an $M \times N$ matrix $\mathbf{F}$ whose entries are independent zero-mean complex (or real) random variables with variance $\frac{1}{N}$ and fourth moments of order $O\left(\frac{1}{N^{2}}\right)$. As $M, N \rightarrow \infty$ with $\frac{N}{M} \rightarrow \beta$, the empirical distribution of the eigenvalues of $\frac{1}{N} \mathbf{F F}^{H}$ converges almost surely to a non-random limiting distribution with density given by:

$$
f_{\beta}(\lambda)=(1-\beta)^{+} \delta(\lambda)+\frac{\sqrt{(\lambda-a)^{+}(b-\lambda)^{+}}}{2 \pi \beta \lambda},
$$

where $a=(1-\sqrt{\beta})^{2}, b=(1+\sqrt{\beta})^{2}, \delta($.$) is a Dirac delta$ function and $(1-\beta)^{+} \delta(\lambda)$ represents the cardinality of zero eigenvalues which can occur if $M>N$. The parameters $a$ and $b$ define the support of the distribution and correspond to $\lambda_{\min }$ and $\lambda_{\max }$ respectively and the ratio $b / a$ defines the $\mathrm{SCN}$ of $\frac{1}{N} \mathbf{F F}^{H}$. The above limiting distribution is the MP law with ratio index $\beta$.

Remark 5.1: In practice, we can have only a finite number of samples and the sample covariance matrix $\hat{\mathbf{R}}_{\mathbf{Y}}(N)$ may deviate from the covariance matrix $\mathbf{R}_{\mathbf{Y}}$ [8]. The eigenvalue distribution of $\hat{\mathbf{R}}_{\mathbf{Y}}(N)$ becomes complicated due to requirement of consideration of finite parameters in the analysis. This makes the choice of the threshold difficult for SS purpose and the performance of SS algorithms becomes sensitive to the choice of threshold at low values of SNR. Although various TW approaches have been proposed in [8] and [22] for accounting the random nature of SCN of finite matrices, we are interested in analyzing the correlation effect on MP based asymptotic methods in this paper. We consider asymptotic analysis in this paper as a first step towards analyzing the effect of noise correlation on sensing performance since asymptotic analysis provides less complex solution than finite analysis and it is more tractable [34]. Furthermore, it can be noted in [16] and [34] that the asymptotic analysis provides good 
approximation to the finite analysis even with a small number of samples.

In this noise only case, $\hat{\mathbf{R}}_{\mathbf{Y}}(N)$ becomes equal to $\hat{\mathbf{R}}_{\hat{\mathbf{Z}}}(N)$ and can be written as:

$$
\hat{\mathbf{R}}_{\mathbf{Y}}(N)=\hat{\mathbf{R}}_{\hat{\mathbf{Z}}}(N)=\boldsymbol{\Theta}^{1 / 2} \mathbf{Z Z} \mathbf{Z}^{H} \boldsymbol{\Theta}^{1 / 2} .
$$

It can be noted that $\hat{\mathbf{R}}_{\mathbf{Y}}(N)$ converges to $\mathbf{R}_{\mathbf{Y}}$ for $N \rightarrow \infty$ [22] and asymptotic analysis still holds true for large values of $N$ [16]. Furthermore, $\hat{\mathbf{R}}_{\mathbf{Z}}(N)=\frac{1}{N} \mathbf{Z} \mathbf{Z}^{H}$ is nearly a Wishart random matrix [11] in white noise scenarios but is no longer a Wishart random matrix in correlated noise scenarios.

To calculate the threshold for SS purpose, we need the support of a.e.p.d.f. of $\mathbf{Y}$, namely, $\lambda_{\max }$ and $\lambda_{\min }$. Due to noncommutative nature of random matrices, it is not straightforward to calculate the eigenvalue distribution of $\mathbf{Y}$ by knowing the eigenvalue distribution of $\boldsymbol{\Theta}$ and $\mathbf{Z}$. Using free probability analysis, the asymptotic spectrum of the product or sum can be obtained from the individual asymptotic spectra without involving the structure of the eigenvectors of the matrices under a asymptotic freeness condition [11]. The asymptotic eigenvalue distribution of $\frac{1}{N} \mathbf{Y} \mathbf{Y}^{H}$ in this context can be obtained by applying multiplicative free convolution property of $\Sigma$ transform in the following way [38].

$$
\Sigma_{\hat{\mathbf{R}}_{\mathbf{Y}}}(z)=\Sigma_{\boldsymbol{\Theta}}(z) \cdot \Sigma_{\hat{\mathbf{R}}_{\mathbf{Z}}}(z)
$$

where $\Sigma_{\Theta}$ and $\Sigma_{\hat{\mathbf{R}}_{Z}}$ are the $\boldsymbol{\Sigma}$ transforms of the densities of eigenvalues of $\Theta$ and $\hat{\mathbf{R}}_{\mathbf{Z}}$ respectively. Since $\Theta$ is a square matrix, $\boldsymbol{\Theta}^{1 / 2} \mathbf{Z} \mathbf{Z}^{H} \boldsymbol{\Theta}^{1 / 2}$ and $\boldsymbol{\Theta} \mathbf{Z} \mathbf{Z}^{H}$ have identical eigenvalues [11]. Since $\hat{\mathbf{R}}_{\mathbf{Z}}$ follows the MP law, its $\Sigma$ transform is given by (33) and then the $\Sigma$ transform of $\hat{\mathbf{R}}_{\mathbf{Y}}$ can be written as:

$$
\Sigma_{\hat{\mathbf{R}}_{\mathbf{Y}}}(z)=\Sigma_{\boldsymbol{\Theta}}(z) \frac{1}{z+\beta} .
$$

The $\Sigma$ transform of corresponding asymptotic eigenvalue distribution $\Sigma_{\Theta}(z)$ can be obtained by choosing a proper model for noise correlation. The asymptotic density of eigenvalues of $\Theta$ can be described as a tilted semicircular law [35], which is a close approximation for the exponential model and is analytically tractable. This density can be described using the following theorem.

Theorem 5.2: [35] Let $\Theta$ be a positive definite matrix which is normalized as: $(1 / M) \operatorname{trace}\{\boldsymbol{\Theta}\}=1$, and whose asymptotic spectrum has the p.d.f.

$$
f_{\Theta}(\lambda)=\frac{1}{2 \pi \mu \lambda^{2}} \sqrt{\left(\frac{\lambda}{\sigma_{1}}-1\right)\left(1-\frac{\lambda}{\sigma_{2}}\right)}
$$

with $\sigma_{1} \leq \lambda \leq \sigma_{2}$ and $\mu=\frac{\left(\sqrt{\sigma_{2}}-\sqrt{\sigma_{1}}\right)^{2}}{4 \sigma_{1} \sigma_{2}}$. If $\mathbf{F}$ is an $M \times N$ standard complex Gaussian matrix as defined in Theorem 5.1, then as $M, N \rightarrow \infty$ with $\frac{N}{M} \rightarrow \beta$, the asymptotic distribution of $\mathbf{W}=\boldsymbol{\Theta}^{1 / 2} \mathbf{F} \mathbf{F}^{H} \boldsymbol{\Theta}^{1 / 2}$ has the following p.d.f.

$$
f_{\mathbf{W}}(\lambda)=(1-\beta)^{+} \delta(\lambda)+\frac{\sqrt{(\lambda-\tilde{a})^{+}(\tilde{b}-\lambda)^{+}}}{2 \pi \lambda(1+\lambda \mu)},
$$

where

$$
\begin{aligned}
& \tilde{a}=1+\beta+2 \mu \beta-2 \sqrt{\beta} \sqrt{(1+\mu)(1+\mu \beta)} \\
& \tilde{b}=1+\beta+2 \mu \beta+2 \sqrt{\beta} \sqrt{(1+\mu)(1+\mu \beta)}
\end{aligned}
$$

The parameters $\tilde{a}$ and $\tilde{b}$ correspond to $\lambda_{\min }$ and $\lambda_{\max }$ respectively and the ratio $\tilde{b} / \tilde{a}$ defines the $\mathrm{SCN}$ of $\mathbf{W}$.

The eigenvalue spread of $\Theta$ is related to the degree of noise correlation i.e., a zero eigenvalue spread corresponds to a zero-correlation model $\Theta=\mathbf{I}_{M}$ and higher spreads are associated with higher correlation modes. In (14), the parameter $\mu$ controls the degree of noise correlation and varies the support of the distribution i.e., for $\mu=0, \tilde{a}=a$ and $\tilde{b}=b$. For the exponential correlation model as stated in [35], the parameter $\mu$ is related to correlation coefficient $\rho$ with the following relation: $\mu=\frac{\rho^{2}}{1-\rho^{2}}$. Furthermore, the SCN is related to $\rho$ with the relation $\mathrm{SCN}=\frac{1+\rho}{1-\rho}$. To calculate $\mu$ in a practical cognitive receiver, the value of $\rho$ can be determined from FS rate based on some empirical model constructed from measurements. In our results, we employ a simple linear model to study the effect of noise correlation as the FS rate increases (see Section VII C).

It can be noted that MP law can be used as a theoretical prediction under the $\mathbb{H}_{0}$ hypothesis with white noise [16]. The support of the eigenvalues of the sample covariance matrix under the $\mathbb{H}_{0}$ hypothesis is finite independently of the distribution of the noise. To decide the absence or presence of signal under white noise scenario, the deviations of distribution of eigenvalues from the normal bounds $a$ and $b$ of MP law can be used. If the eigenvalues appear outside these bounds, then it can be decided that there is presence of PU signal and if all the eigenvalues lie within the bounds of MP law, it can be decided that there is absence of PU signal. In case of noise correlation, the bounds of eigenvalue distribution of sample covariance matrix become different than the bounds obtained in white noise scenarios and MP law no longer applies. The new bounds $(\tilde{a}, \tilde{b})$ depend on the noise correlation parameter $\mu$. We present the sensing example with new bounds for FS scenario in the following subsection.

\section{A. Sensing With FS}

The parameter $\mu$ depends on the sampling rate applied in the receiver since noise correlation increases along with the sampling rate. Sampling rate can be varied from the symbol rate to some order of the symbol rate and the effect of sampling rate on sensing performance can be evaluated by varying the correlation level. Let us consider that both noise distribution and noise variance are unknown to the detector to reflect the practical scenario. It can be noted that value of the SCN under the $\mathbb{H}_{0}$ hypothesis does not depend on the noise variance. Under white noise scenario, the decision statistic for MP law can be calculated as [16]:

$$
\text { decision }= \begin{cases}\mathbb{H}_{0}, & \text { if } \mathrm{SCN} \leq \frac{b}{a} \\ \mathbb{H}_{1}, & \text { otherwise }\end{cases}
$$

It can be noted that the values of the supports $a$ and $b$ can be determined from the closed form of the a.e.p.d.f. provided in Theorem 5.1. More specifically, the values of these supports can be calculated by finding out the crossing points of the a.e.p.d.f. with the $\mathrm{x}$-axis. Similar analysis can be applied for sensing in presence of the correlated noise. Equation (14) from 
Theorem 5.2 provides the a.e.p.d.f. for the received signal's covariance matrix in presence of noise correlation. By finding out the crossing points of this a.e.p.d.f. with the $\mathrm{x}$-axis, the supports $\tilde{a}$ and $\tilde{b}$ of eigenvalue distribution in presence of noise correlation can be determined. Then the decision about the presence or absence of the PU signal under correlated noise scenarios can be made on the basis of the following condition:

$$
\text { decision }= \begin{cases}\mathbb{H}_{0}, & \text { if } \mathrm{SCN} \leq \frac{\tilde{b}}{\tilde{a}} \\ \mathbb{H}_{1}, & \text { otherwise }\end{cases}
$$

When FS rate $M$ is applied at the CR, $M$ rows of sample covariance matrix become correlated. Since the value of $\rho$ varies from 0 to 1 , the relation between the FS rate $M$ and the correlation coefficient $\rho$ is considered as a simple linear model ${ }^{6}$ as shown below

$$
\rho=\varepsilon\left(\frac{1}{\beta}-\frac{1}{N}\right)
$$

where $\varepsilon$ is a parameter defining the slope of the linear dependence. The above equation provides a linear relation between $\rho$ and $M$. Since $\beta=\frac{N}{M}, \rho=0$ for $M=1$ i.e., symbol rate sampling and $\rho=1$ for $M=\frac{N}{\varepsilon}+1$. Equation (18) is a specification of the model used in $(8)$ and is used to jointly alter the level of the correlation with the FS rate.

\section{ANALYSIS UNDER $\mathbb{H}_{1}$ HYPOTHESIS}

\section{A. White Noise}

Assuming that signal and noise are independent, for very large value of $N$, (5) leads to the following approximation for the white noise scenario [8].

$$
\lim _{N \rightarrow \infty} \hat{\mathbf{R}}_{\mathbf{Y}}(N) \approx p \mathbf{H} \mathbf{H}^{H}+\hat{\mathbf{R}}_{\mathbf{Z}}
$$

In this scenario, the sample covariance of received signal under assumed conditions can be realized as the sum of two Wishart matrices i.e., $p \hat{\mathbf{R}}_{\mathbf{H}}=p \mathbf{H} \mathbf{H}^{H}$ and $\hat{\mathbf{R}}_{\mathbf{Z}}$ with same degree of freedom and different covariance structures. In this condition, MP law holds true for both matrices. Although it is possible to find another Wishart matrix from the the addition of $p \hat{\mathbf{R}}_{\mathbf{H}}$ and $\hat{\mathbf{R}}_{\mathbf{Z}}$ approximately (see Lemma 6, [39]) and then apply scaled MP law by scaling with variance $\left(1+p^{2}\right)$ for the new Wishart matrix, we use free probability theory for more accurate analysis. The $\mathrm{R}$ transform of eigenvalue density function of $\hat{\mathbf{R}}_{\mathbf{Y}}$ can be found by adding the $\mathrm{R}$ transforms of density functions of $p \hat{\mathbf{R}}_{\mathbf{H}}$ and $\hat{\mathbf{R}}_{\mathbf{Z}}$ using free probability theory. Using (32), the $\mathbf{R}$ transform of $p \hat{\mathbf{R}}_{\mathbf{H}}$ can be written as:

$$
\mathcal{R}_{p \hat{\mathbf{R}}_{\mathbf{H}}}(z)=p \mathcal{R}_{\hat{\mathbf{R}}_{\mathbf{H}}}(p z)=\frac{p \beta}{1-p z} .
$$

Since the $\mathbf{R}$ transform of $\hat{\mathbf{R}}_{\mathbf{Z}}$ is $\mathcal{R}_{\hat{\mathbf{R}}_{\mathbf{Z}}}(z)=\frac{\beta}{1-z}$ from (31), the combined $\mathrm{R}$ transform can be written as:

$$
\mathcal{R}_{\hat{\mathbf{R}}_{\mathbf{Y}}}(z)=\frac{p \beta}{1-p z}+\frac{\beta}{1-z} .
$$

\footnotetext{
${ }^{6}$ This is a simple analytical example and the same method can be applied to more exact relation models which can be acquired through measurements on the CR equipment.
}

The inverse Stieltjes transform can be obtained by applying (21) on (30). Then the Stieltjes transform $\mathcal{S}_{\hat{\mathbf{R}}_{\mathbf{Y}}}$ of the asymptotic distribution of $\frac{1}{N} \mathbf{Y} \mathbf{Y}^{H}$ under white noise scenarios can be obtained for any $z \in \mathbb{C}$ by solving the following cubic polymonial ${ }^{7}$.

$$
\begin{array}{r}
(p z) \mathcal{S}_{\hat{\mathbf{R}}_{\mathbf{Y}}}^{3}(z)+(p(-2 \beta+z+1)+z) \mathcal{S}_{\hat{\mathbf{R}}_{\mathbf{Y}}}^{2}(z) \\
+((1-\beta)(1+p)+z) \mathcal{S}_{\hat{\mathbf{R}}_{\mathbf{Y}}}(z)+1 .
\end{array}
$$

Then the a.e.p.d.f. of $\hat{\mathbf{R}}_{\mathbf{Y}}$ under $\mathbb{H}_{0}$ hypothesis in the presence of white noise is obtained by determining the imaginary part of the Stieltjes transform $\mathcal{S}_{\hat{\mathbf{R}}_{\mathbf{Y}}}$ for real arguments in the following way.

$$
f_{\mathbf{Y}}^{\infty}=\lim _{y \rightarrow 0^{+}} \frac{1}{\pi} \operatorname{Im}\left\{\mathcal{S}_{\hat{\mathbf{R}}_{\mathbf{Y}}}(x+j y)\right\} .
$$

\section{B. Correlated Noise}

Using the similar arguments as in the above subsection, the following approximation can be written for the correlated noise scenario.

$$
\lim _{N \rightarrow \infty} \hat{\mathbf{R}}_{\mathbf{Y}}(N) \approx p \mathbf{H} \mathbf{H}^{H}+\hat{\mathbf{R}}_{\hat{\mathbf{Z}}}
$$

In correlated noise scenarios, the sample covariance of received signal under assumed conditions can be realized as a sum of one Wishart matrix i.e., $p \hat{\mathbf{R}}_{\mathbf{H}}$ and another correlated Wishart matrix $\hat{\mathbf{R}}_{\hat{\mathbf{z}}}$. In this condition, MP law can be applied for $p \hat{\mathbf{R}}_{\mathbf{H}}$ and the analysis carried out under $\mathbb{H}_{0}$ hypothesis in Section $\mathrm{V}$ can be applied for $\hat{\mathbf{R}}_{\hat{\mathbf{Z}}}$. Then the $\mathrm{R}$ transform of density function of the received signal can be found by adding the $\mathrm{R}$ transforms of density functions of $p \hat{\mathbf{R}}_{\mathbf{H}}$ and $\hat{\mathbf{R}}_{\hat{\mathbf{Z}}}$. The Stieltjes transform of $\hat{\mathbf{R}}_{\hat{\mathbf{Z}}}$ can be written as [35]:

$\mathcal{S}_{\hat{\mathbf{R}}_{\mathbf{z}}}(z)=\frac{z+2 z \mu+1-\beta+\sqrt{[z-(1+\beta)]^{2}-4 \beta(1+\mu z)}}{2 z(1+\mu z)}$.

Then the R transform for $\hat{\mathbf{R}}_{\hat{\mathbf{Z}}}$ is calculated using (30) and can be expressed as:

$$
\mathcal{R}_{\hat{\mathbf{R}}_{\hat{\mathbf{Z}}}}(z)=-\frac{1}{2} \frac{\left(z-1+\sqrt{\left(z^{2}-2 z+1-4 \mu \beta z\right)}\right)}{\mu z} .
$$

The combined $\mathrm{R}$ transform then becomes

$\mathcal{R}_{\hat{\mathbf{R}}_{\mathbf{Y}}}(z)=\frac{p \beta}{(1-p z)}-\frac{1}{2} \frac{\left(-1+z+\sqrt{\left(1-2 z+z^{2}-4 \mu \beta z\right)}\right)}{z \mu}$.

The inverse Stieltjes transform can be obtained by applying (27) on (30). Then the Stieltjes transform $\mathcal{S}_{\hat{\mathbf{R}}_{\mathbf{Y}}}$ of the asymptotic distribution of $\frac{1}{N} \mathbf{Y Y}^{H}$ under correlated noise scenarios can be obtained for any $z \in \mathbb{C}$ by solving the quartic polymonial (28).

Then the a.e.p.d.f. of $\hat{\mathbf{R}}_{\mathbf{Y}}$ under $\mathbb{H}_{1}$ hypothesis in the presence of correlated noise can be found using (23).

Remark 6.1: We can find the roots of the polymonials (22) and (28) in closed forms. The closed forms are not specifically written in this paper because the solution includes many terms which provide no further insight. In practice, we can just solve these polymonials with a mathematical software for finding the Stieltjes transforms under the considered scenarios.

\footnotetext{
${ }^{7}$ We select the imaginary root which complies with the definition and properties of Stieltjes transform (see Appendix).
} 


$$
\begin{gathered}
\left(z p^{2}(1+\mu z)\right) \mathcal{S}_{\hat{\mathbf{R}}_{\mathbf{Y}}}^{4}(z)+\left(2 z \mu p(z-p \beta)+p^{2}(1+2 z \mu+z-2 \beta)+2 z p\right) \mathcal{S}_{\hat{\mathbf{R}}_{\mathbf{Y}}}^{3}(z)+\left(p^{2}\left(\mu(1-\beta)^{2}+1-\beta\right)+2 p\right. \\
\left.(1+z+\mu z(2-\beta))+z-3 p \beta+z^{2} \mu\right) \mathcal{S}_{\hat{\mathbf{R}}_{\mathbf{Y}}}^{2}(z)+(2 p(1+\mu(1-\beta))+z(1+2 \mu)-\beta(1+p)+1) \mathcal{S}_{\hat{\mathbf{R}}_{\mathbf{Y}}}(z)+1+\mu
\end{gathered}
$$

\section{Proposed SNR Estimation Technique}

The SNR estimation is carried out based on the support of a.e.p.d.f. of the received signal's covariance matrix under both white noise and correlated noise scenarios. The support of a.e.p.d.f. of $\frac{1}{N} \mathbf{Y} \mathbf{Y}^{H}$ under correlated noise is calculated based on (28) and under white noise based on (22). It can be noted from the polymonials (22) and (28) that we have a connection between $\lambda_{\max }$ and the SNR. Since we know the value of $\beta$ and we can measure the value of $\rho$ by carrying out measurements at the $\mathrm{CR}$ equipment, we can estimate the value of $p$ by sensing the maximum eigenvalue of $\frac{1}{N} \mathbf{Y} \mathbf{Y}^{H}$. Lookup tables are provided for convenience in order to estimate the SNR of the PU signal (see Section VII B). We consider the following three cases: (i) signal plus correlated noise, (ii) correlated noise only, and (iii) signal plus white noise. In the lookup table, we present the maximum eigenvalues of the received signal's covariance matrix for above three cases for different values of SNR and $\beta$. We can estimate the received SNR of the PU signal based on the $\lambda_{\max }$ in the following way. Firstly, we develop the SNR estimation table (Table I) based on (22) for white noise scenario and based on (28) for correlated noise scenario. By using the proposed sensing model, we can find the value of $\lambda_{\max }$ and then by looking into the table which provides the value of $\lambda_{\max }$ for a certain value of SNR, we can find the SNR corresponding to a particular $\lambda_{\max }$. The parameters $\beta$ and $\rho$ are assumed known as operating parameters of the sensing module. Based on this estimated SNR, we could potentially design suitable underlay transmission strategies for secondary transmission in the considered scenario with short range primary and secondary wireless systems as mentioned in Section I. In Section VII, we provide the normalized MSE versus SNR plot (see Fig. 11) to evaluate the performance of this estimation technique.

\section{Numerical RESUlts}

In this section, we study the performance of eigenvalue based sensing in the presence of noise correlation with the proposed decision bounds. We use probability of correct decision as a sensing performance metric as mentioned in Section III and define this metric in the following way. Let $P\left(\mathbb{H}_{i} ; \mathbb{H}_{j}\right)$ indicate the probability of deciding hypothesis $\mathbb{H}_{i}$ when hypothesis $\mathbb{H}_{j}$ is true with $\{i, j\} \in\{0,1\}$. The probability of detection $\left(P_{d}\right)$ can be defined as: $P_{d}=P\left(\mathbb{H}_{1} ; \mathbb{H}_{1}\right)$ and the $P_{f}$ can be defined as: $P_{f}=P\left(\mathbb{H}_{1} ; \mathbb{H}_{0}\right)$ [40]. Then the probability of correct decision is defined as: $\left(P\left(\mathbb{H}_{1} ; \mathbb{H}_{1}\right)+P\left(\mathbb{H}_{0} ; \mathbb{H}_{0}\right)\right) / 2$ i.e., $P_{d}+\left(1-P_{f}\right) / 2$. In other words, it depicts how many correct decisions are made out of the total considered iterations under both hypotheses ${ }^{8}$. In the presented simulation results,

\footnotetext{
${ }^{8}$ Since threshold is fixed in our scenario and noise correlation affects the value of $P_{f}$, we consider number of correct decisions under both hypotheses.
}

$10^{3}$ iterations were considered. We consider Rayleigh fading channel in our simulation model and its coefficients are generated from random complex numbers whose real and imaginary components are i.i.d. Gaussian variables. As a result, the channel matrix $\mathbf{H}$ is a Gaussian matrix i.e., $\mathbf{H} \sim \mathcal{C N}(0, \mathbf{I})$.

Furthermore, we present an SNR estimation method under $\mathbb{H}_{1}$ hypothesis. The normalized MSE is considered as a parameter to characterize the performance of the proposed SNR estimation technique and is defined as:

$$
\mathrm{MSE}=\frac{\mathbb{E}[\hat{p}-p]^{2}}{p^{2}}
$$

where $\hat{p}$ is the estimated SNR with the proposed method and $p$ is the actual SNR.

\section{A. Eigenvalue Based SS}

The performance of the proposed sensing scheme has been analyzed in white and correlated noise scenarios. In case of white noise scenarios, it has been noted that the eigenvalue distribution of the received signal's covariance matrix follows the MP law and the distribution is limited to the bounds given by this law. Therefore, the decision rule in (16) is used for sensing of the PU signal under white noise scenarios. However, in the presence of noise correlation, the eigenvalue distribution deviates from the distribution under white noise scenario (Fig. 2 , [37]) and new decision rule proposed in (17) is considered.

To compare the sensing performance with MP based threshold and new proposed threshold, the probability of correct decision versus SNR for $\rho=0.5, \beta=1 / 6, N=60$ is depicted in Fig. 3. It can be observed that sensing with (17) outperforms than sensing with (16) in correlated noise scenarios. Figure 4 shows the sensing performance versus correlation coefficient at SNR value of $-6 \mathrm{~dB}$ and $\beta=1 / 6$ and it can be noted that with the increased amount of noise correlation, the sensing with MP bounds decreases drastically and sensing with (17) gives better performance up to some value of correlation. Moreover, it has been noted that new bounds also do not provide better sensing at high correlation region. This is due to the fact that the threshold increases and the asymptotic eigenvalue support of $\mathbb{H}_{1}$ is subsumed in the one of $\mathbb{H}_{0}$ at this region.

Figure 5 depicts the probability of a false alarm versus correlation coefficient for SNR $=-6 \mathrm{~dB}, \beta=1 / 6, N=60$. It can be noted that the value of $P_{f}$ differs for sensing in white noise and correlated noise scenarios. The value of $P_{f}$ is very small for sensing with (16) in white noise scenarios but it varies with the value of $\rho$ for sensing in correlated noise scenarios. In correlated noise scenarios, the value of $P_{f}$ with the increase in the value of $\rho$ becomes worse for sensing with (16) than for sensing with (17). This has been further illustrated by the plots of CDF curves and thresholds in Fig. 6. From these results, it can be noted that overall sensing 


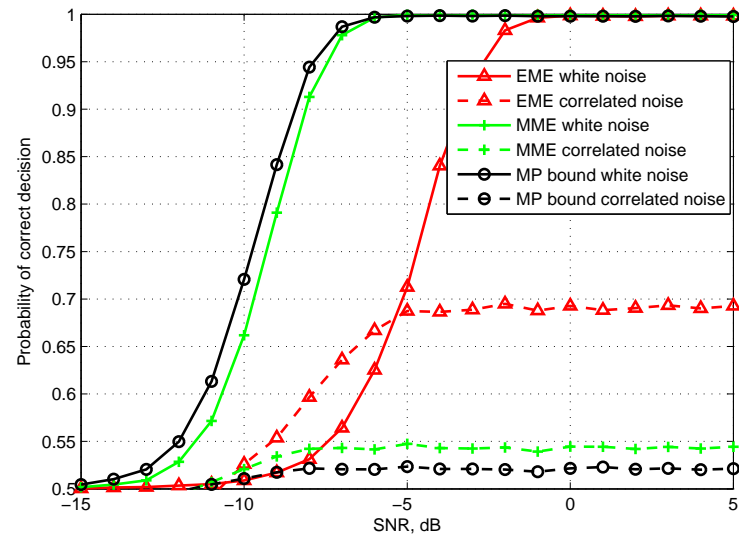

Fig. 2: Sensing performance of different methods in white and correlated noise scenarios $(\beta=1 / 8, \rho=0.6, N=80)$

performance is improved with the proposed bounds since we get lower $P_{f}$ while sensing with the proposed bounds than with the MP bounds up to a certain level of correlation. The CDF curves for white noise and correlated noise scenarios in Fig. 6 were plotted by accumulating the decision statistics over $10^{3}$ iterations for $\beta=1 / 6, \rho=0.5, N=60$. Figure 7 shows the probability of detection versus SNR for $P_{f}=0.004$ in white noise and correlated noise scenarios. The $P_{f}$ value for white noise scenarios was noted to be 0.004 during simulation as reflected in Fig. 5 and Fig. 6. To plot $P_{d}$ versus SNR for the same value of $P_{f}$, the decision threshold in correlated noise scenarios was adjusted to make the $P_{f}$ value equal to its value in white noise scenarios numerically using the CDF curves of the decision statistics shown in Fig. 6.

Analysis under $\mathbb{H}_{1}$ hypothesis case was considered by taking the combination of signal and noise under both scenarios. Figure 8 (a) shows the theoretical and simulated eigenvalue distribution of covariance matrix of received signal i.e., $\frac{1}{N} \mathbf{Y} \mathbf{Y}^{H}$ for $\mathrm{SNR}=-2 \mathrm{~dB}$ and $\beta=1$ under white noise scenarios. The histograms of the eigenvalues were created by accumulating the eigenvalues over $10^{3}$ iterations. The theoretical result was obtained by evaluating the polynomial given by (22). Similarly, Fig. 8 (b) shows the eigenvalue distribution of covariance matrix of received signal for $\mathrm{SNR}=-2 \mathrm{~dB}$, $\mathrm{SCN}=3$ and $\beta=1$ under correlated noise. In this case, theoretical result was obtained by evaluating the polynomial given by (28). From the figure 8 , it can be observed that the theoretical and simulated density functions perfectly match.

To observe the variation of of received signal's covariance matrix with respect to SNR, we present the maximum eigenvalue versus SNR plot in Fig. 9 for both correlated and white noise scenarios. From the figure, it can be observed that the maximum eigenvalue has higher value in correlated scenario than in white noise scenario over the considered range of SNR (from $-10 \mathrm{~dB}$ to $2 \mathrm{~dB}$ ) and the gap between these two curves goes on decreasing while increasing the value of SNR. Figure 10 shows the plot of the maximum eigenvalue of the received signal's covariance matrix versus SCN of correlation matrix for the following three cases: (i) signal plus correlated noise, (ii) correlated noise only, and (iii) signal plus white noise. It can be observed that the maximum eigenvalue for the first case

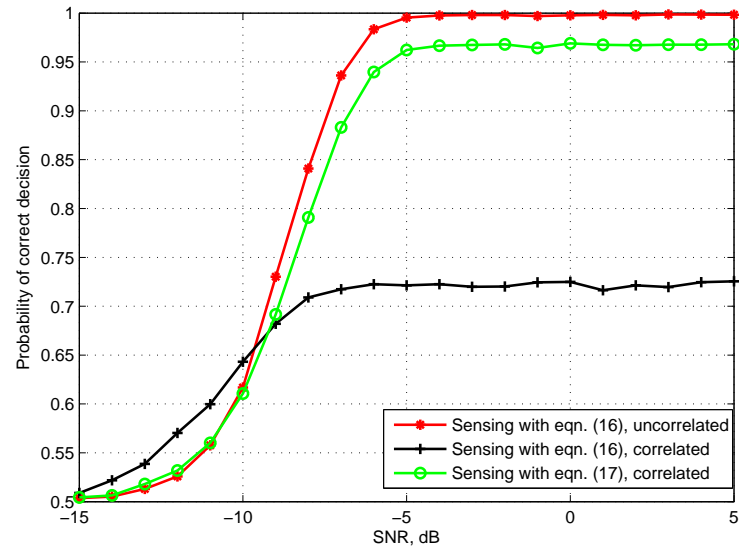

Fig. 3: Sensing performance versus SNR with (16) and (17) $(\beta=1 / 6, \rho=0.5, N=60)$

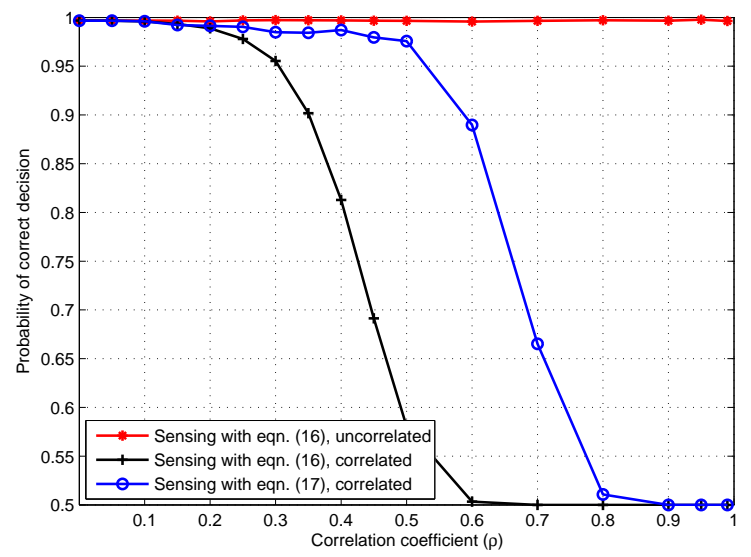

Fig. 4: Sensing performance versus correlation coefficient $(\mathrm{SNR}=-6$ $\mathrm{dB}, \beta=1 / 6, N=60)$

is greater than the maximum eigenvalue in the second case and the difference remains more or less consistent for all the considered values of SCN (from 2 to 20). With respect to the white noise scenarios, the maximum eigenvalue in correlated noise scenarios increases almost linearly with the value of SCN.

\section{B. SNR Estimation}

Table I shows the lookup table for different values of SCNs of the correlation matrix. This table can be used to estimate the SNR of the PU signal based on the values of SCN and $\beta$ for both correlated and white noise scenarios. The value of SCN can be derived from the measurements of $\rho$ as mentioned in Section V. For example, if the value of $\mathrm{SCN}$ is $3, \beta$ is 1 and the maximum eigenvalue of covariance matrix of received signal i.e., $\frac{1}{N} \mathbf{Y Y}^{H}$ is 5.75 in signal plus correlated noise case, we can estimate that SNR of the PU signal is $0 \mathrm{~dB}$ and intermediate values can be calculated through interpolation. From the table, it can be observed that at lower SNR values, the difference in the maximum eigenvalue of signal plus correlated noise case and correlated noise only case becomes very small and it becomes difficult to distinguish signal from the noise.

Figure 11 shows the normalized MSE versus SNR plot for white noise and correlated noise scenarios for different 


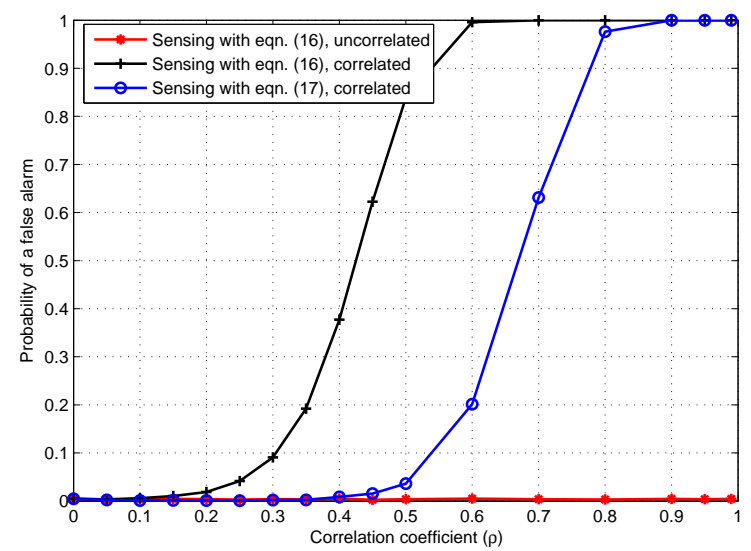

Fig. 5: Probability of a false alarm versus correlation coefficient $(\mathrm{SNR}=-6 \mathrm{~dB}, \beta=1 / 6, N=60)$

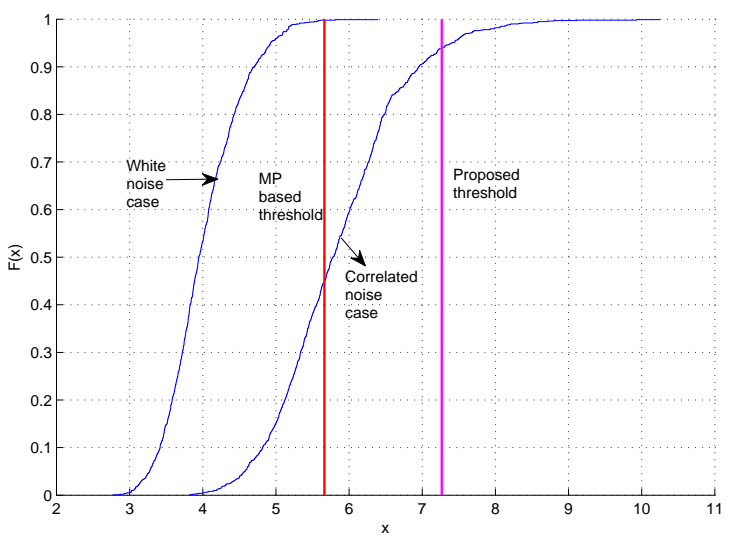

Fig. 6: Cumulative distribution functions of decision statistics under $\mathbb{H}_{0}$ hypothesis and thresholds for white and correlated noise scenarios $(\beta=1 / 6, \rho=0.5, N=60)$



Fig. 7: Probability of detection versus SNR in white and correlated noise scenarios for $P_{f}=0.004(\beta=1 / 6, \rho=0.5, N=60)$

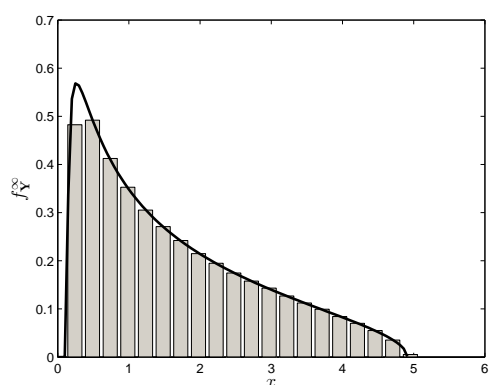

(a) $\mathbf{Y}=\left(\mathbf{H S}_{d}+\mathbf{Z}\right)$



(b) $\mathbf{Y}=\left(\mathbf{H S}_{d}+\hat{\mathbf{Z}}\right)$

Fig. 8: Theoretical and simulated eigenvalue distribution of received signal for different cases (SNR $=-2 \mathrm{~dB}, \beta=1, N=50$ ): (a) signal plus white noise, (b) signal plus correlated noise $(S C N=3)$

SCNs of the noise correlation matrix. From the figure, it can be observed that for all considered cases, normalized MSE decreases with the SNR. In case of white noise scenario, we can estimate the PU SNR with less than $0.5 \%$ normalized MSE up to $0 \mathrm{~dB}$ and with less than $1 \%$ normalized MSE up to $-1 \mathrm{~dB}$. Similarly, in case of correlated noise scenarios, we can estimate the SNR with less than $1 \%$ normalized MSE up to $0 \mathrm{~dB}$ for all considered values of SCN, with less than $2 \%$ normalized MSE up to $-1 \mathrm{~dB}$ and after SNR values of $3 \mathrm{~dB}, \mathrm{SNR}$ in all the cases can be estimated with almost 0.2 $\%$ normalized MSE. Furthermore, it can be noted that the normalized MSE performance decreases with the increase in the value of SCN at lower SNR values and it becomes almost stable if we go to higher SNR values beyond $3 \mathrm{~dB}$. From this result, it can be concluded that the proposed technique can be used to estimate the PU SNR reliably in the presence of correlated noise and noise correlation mostly affects the SNR estimation performance at lower SNR values.

\section{FS Operating Point}

Figure 12 shows the probability of correct decision versus FS rate for $\varepsilon=3.5$. The FS rate has been increased from 1 to 11 and noise correlation has been calculated using (18) for different values of $M$. It can be noted that the sensing performance increases with the FS rate for white noise scenario. However, at the same time, noise becomes correlated due to 
This article has been accepted for publication in a future issue of this journal, but has not been fully edited. Content may change prior to final publication. IEEE TRANSACTIONS ON VEHICULAR TECHNOLOGY, ACCEPTED FOR PUBLICATION

TABLE I: Lookup table for the proposed SNR estimation technique

\begin{tabular}{l|c|c|c|c|c} 
SCN & $\beta$ & SNR (dB) & $\begin{array}{c}\text { Signal plus white Noise } \\
\lambda_{\max }\left(\mathbf{H S}_{d}+\mathbf{Z}\right)\end{array}$ & $\begin{array}{c}\text { Correlated Noise } \\
\lambda_{\max }(\mathbf{\mathbf { Z }})\end{array}$ & $\begin{array}{c}\text { Signal plus correlated Noise } \\
\lambda_{\max }\left(\mathbf{H S}_{d}+\mathbf{\mathbf { Z }}\right)\end{array}$ \\
\hline 2 & 1 & 5 & 13.18 & 3.90 & 13.19 \\
2 & 1 & 4 & 10.77 & 3.90 & 10.78 \\
2 & 1 & 2 & 7.45 & 3.90 & 7.47 \\
2 & 1 & 0 & 5.59 & 3.90 & 5.65 \\
2 & 1 & -2 & 4.70 & 3.90 & 4.79 \\
2 & 1 & -4 & 4.29 & 3.90 & 4.38 \\
2 & 1 & -6 & 4.08 & 3.90 & 4.19 \\
2 & 1 & -8 & 3.96 & 3.90 & 4.07 \\
2 & 1 & -10 & 3.90 & 3.90 & 4.01 \\
\hline 3 & 1 & 5 & 13.18 & 4.08 & 13.21 \\
3 & 1 & 4 & 10.77 & 4.08 & 7.52 \\
3 & 1 & 2 & 7.45 & 4.08 & 5.75 \\
3 & 1 & 0 & 5.59 & 4.08 & 4.93 \\
3 & 1 & -2 & 4.70 & 4.08 & 4.55 \\
3 & 1 & -4 & 4.29 & 4.08 & 4.35 \\
3 & 1 & -6 & 4.08 & 4.08 & 4.24 \\
3 & 1 & -8 & 3.96 & 4.08 & 4.18 \\
3 & 1 & -10 & 3.90 & 4.25 & 13.22 \\
4 & 1 & 5 & 13.18 & 4.25 & 10.83 \\
4 & 1 & 4 & 10.77 & 4.25 & 7.57 \\
4 & 1 & 2 & 7.45 & 4.25 & 5.86 \\
4 & 1 & 0 & 5.59 & 4.25 & 5.08 \\
4 & 1 & -2 & 4.70 & 4.25 & 4.72 \\
4 & 1 & -4 & 4.29 & 4.25 & 4.52 \\
4 & 1 & -6 & 4.08 & 4.25 & 4.35 \\
\hline
\end{tabular}

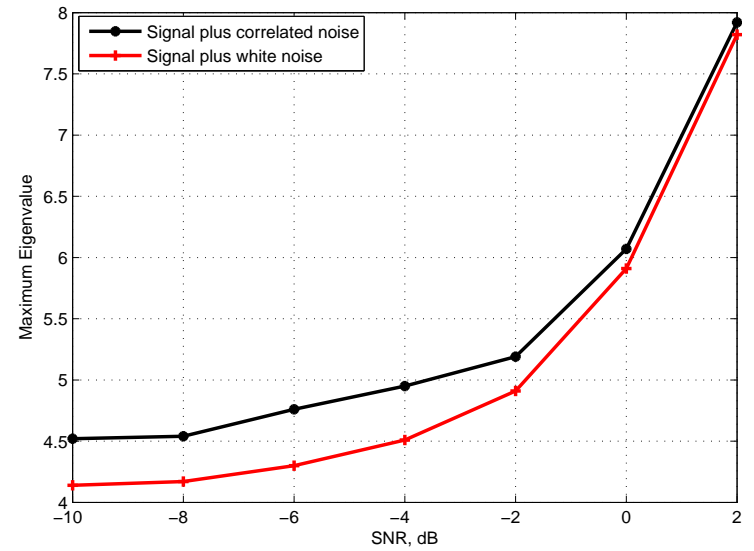

Fig. 9: Maximum eigenvalue versus SNR for correlated and white noise scenarios ( $\mathrm{SCN}=3, \beta=1, N=60)$

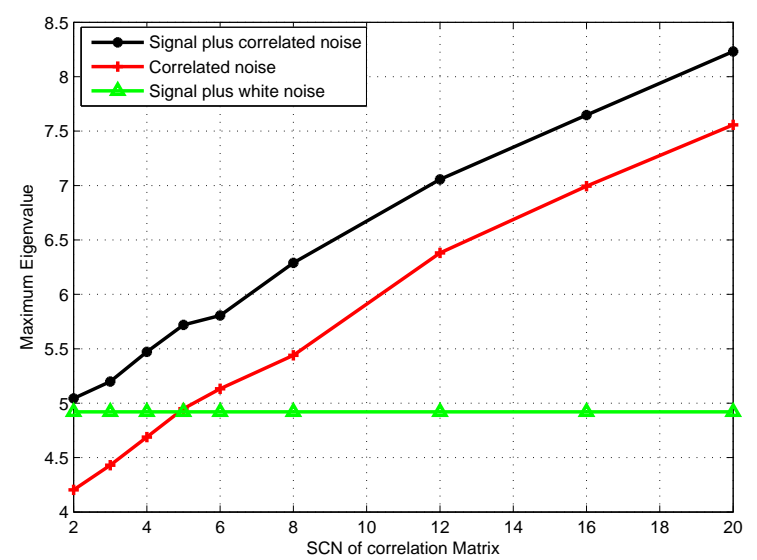

Fig. 10: Maximum eigenvalue versus SCN for correlated and white noise scenarios $(\mathrm{SNR}=-2 \mathrm{~dB}, \beta=1, N=60)$

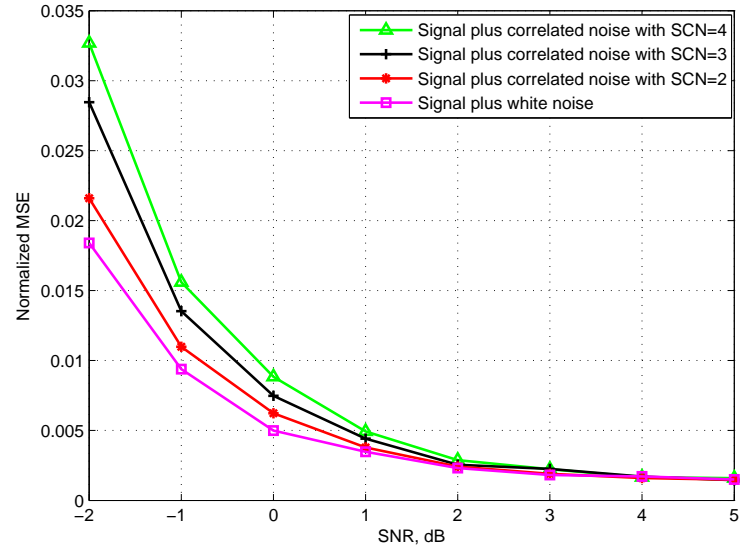

Fig. 11: Normalized MSE versus SNR for SNR estimation using the proposed technique $(N=100, \beta=1)$

FS and increasing the sampling rate does not monotonically increase the performance. From Fig. 12, it can be noted that for $N=60$, SNR $=-5 \mathrm{~dB}$, the performance increases up to FS rate $M=8$ and for $M>8$, the sensing with (17) saturates. It can be observed that increasing sampling rate enhances the sensing performance up to a certain FS rate, however, this also increases the complexity in the receiver. Thus it can be concluded that optimum sampling rate should be chosen at the receiver without increasing further complexity since larger sampling rate does not enhance the performance due to noise correlation.

\section{CONCLUSION AND FUTURE WORK}

In this paper, the performance of eigenvalue based sensing has been analyzed in the presence of noise correlation. This 


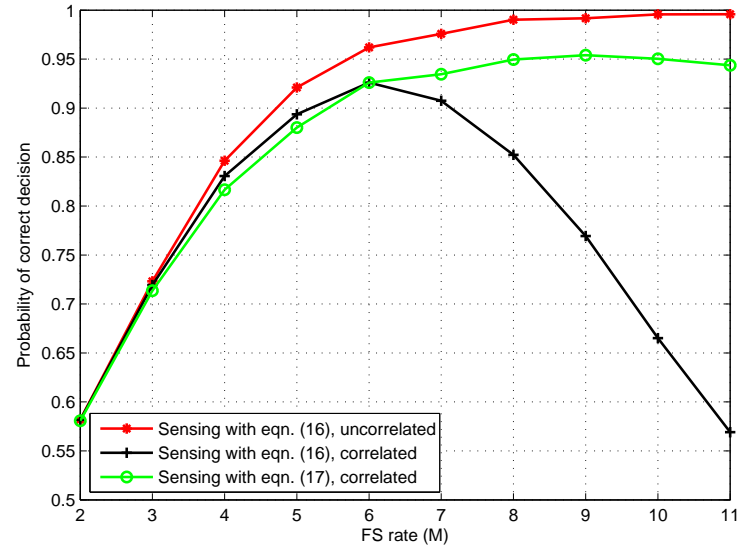

Fig. 12: Sensing performance versus FS rate $(N=60, \mathrm{SNR}=-5 \mathrm{~dB})$

case often appears due to imperfections in filtering or oversampling and results in non-Wishart covariance matrices. A new SCN-based threshold has been proposed for improved sensing in the presence of noise correlation. Furthermore, an SNR estimation technique based on the maximum eigenvalue of the received signal's covariance matrix has been proposed and the performance of the proposed technique has been analyzed with normalized MSE. It has been shown that SNRs up to $0 \mathrm{~dB}$ can be reliably estimated with less than $1 \%$ normalized MSE in the presence of correlated noise without any knowledge of the noise variance. Moreover, the performance of FS based SS technique is studied and it has been noted that SS efficiency increases with the FS rate up to a certain limit and it does not provide performance advantage beyond this limit. Therefore, it can be concluded that an optimal operating point for the FS rate should be selected to maintain a good trade-off between performance and complexity.

For practical implementation of a CR, sensing techniques should work efficiently in the realistic scenarios where noise and channel correlation are always present at some level. Exploring efficient sensing techniques in these scenarios has remained as an open research issue. In our future work, we plan to analyze the effect of channel correlation as well as the combined effect of noise correlation and channel correlation on different SS techniques and propose new sensing schemes suitable for these scenarios.

\section{APPENDIX \\ Random Matrix Theory Preliminaries}

Let $F_{\mathbf{X}}(x)$ be the eigenvalue probability density function of a matrix $\mathbf{X}$.

Theorem 8.1: The Stieltjes transform $\mathcal{S}_{\mathbf{X}}(z)$ of a positive semidefinite matrix $\mathbf{X}$ is defined by [11]:

$$
\mathcal{S}_{\mathbf{X}}(z)=\mathbb{E}\left[\frac{1}{\mathbf{X}-z}\right]=\int_{-\infty}^{\infty} \frac{1}{\lambda-z} d F_{\mathbf{X}}(\lambda)
$$

Theorem 8.2: The $\mathrm{R}$ transform is related to the inverse of Stieltjes transform as [11]:

$$
\mathcal{R}_{\mathbf{X}}(z)=\mathcal{S}_{\mathbf{X}}^{-1}(-z)-\frac{1}{z}
$$

Theorem 8.3: For a Wishart random matrix $\mathbf{X}$, the $\mathrm{R}$ trans- form of the density of eigenvalues of $\mathbf{X}$ is defined as [11]:

$$
\mathcal{R}_{\mathbf{X}}(z)=\frac{\beta}{1-z}
$$

For any $a>0$,

$$
\mathcal{R}_{a \mathbf{X}}=a \mathcal{R}_{\mathbf{X}}(a z)
$$

Theorem 8.4: For a Wishart random matrix $\mathbf{X}$, the $\Sigma$ transform of the density of eigenvalues of $\mathbf{X}$ is defined as [11]:

$$
\Sigma_{\mathbf{X}}(z)=\frac{1}{z+\beta}
$$

\section{ACKNOWLEDGEMENT}

This work was supported by the National Research Fund, Luxembourg under AFR (Aids Training-Research) grant for Ph.D. project (Reference 3069102) on "Spectrum Sensing, Resource Allocation and Resource Management Strategies for Satellite Cognitive Communications", under the CORE project "CO2SAT: Cooperative and Cognitive Architectures for Satellite Networks". The authors would like to thank anonymous reviewers for providing their valuable suggestions.

\section{REFERENCES}

[1] A. Goldsmith, S. Jafar, I. Maric, and S. Srinivasa, "Breaking spectrum gridlock with cognitive radios: An information theoretic perspective," Proc. of the IEEE, vol. 97, no. 5, pp. 894 -914, May 2009.

[2] T. Yucek and H. Arslan, "A survey of spectrum sensing algorithms for cognitive radio applications," IEEE Commun. Surv. Tutor., vol. 11, no. 1, pp. $116-130$, quarter 2009.

[3] R. Tandra and A. Sahai, "SNR walls for signal detection," IEEE J. Sel. Topics Signal Process., vol. 2, no. 1, pp. 4-17, Feb. 2008.

[4] L. Shen, H. Wang, W. Zhang, and Z. Zhao, "Blind spectrum sensing for cognitive radio channels with noise uncertainty," IEEE Trans. Wireless Commun., vol. 10, no. 6, pp. 1721 -1724, June 2011.

[5] M. Orooji, R. Soosahabi, and M. Naraghi-Pour, "Blind spectrum sensing using antenna arrays and path correlation," IEEE Trans. Veh. Technol., vol. 60 , no. 8 , pp. $3758-3767$, Oct. 2011.

[6] S. Bokharaiee, H. Nguyen, and E. Shwedyk, "Blind spectrum sensing for OFDM-based cognitive radio systems," IEEE Trans. Veh. Technol., vol. 60 , no. 3, pp. $858-871$, March 2011 .

[7] P. De and Y.-C. Liang, "Blind spectrum sensing algorithms for cognitive radio networks," IEEE Trans. Veh. Technol., vol. 57, no. 5, pp. 2834 2842, Sept. 2008.

[8] Y. Zeng and Y. chang Liang, "Eigenvalue-based spectrum sensing algorithms for cognitive radio," IEEE Trans. Commun., vol. 57, no. 6, pp. $1784-1793$, June 2009.

[9] W. Zhang, G. Abreu, M. Inamori, and Y. Sanada, "Spectrum sensing algorithms via finite random matrices," IEEE Trans. Commun., vol. 60, no. 1 , pp. $164-175$, Jan. 2012.

[10] A. Kortun, T. Ratnarajah, M. Sellathurai, C. Zhong, and C. Papadias, "On the performance of eigenvalue-based cooperative spectrum sensing for cognitive radio," IEEE J. Sel. Topics Signal Process., vol. 5, no. 1, pp. $49-55$, Feb. 2011.

[11] A. M. Tulino and S. Verdu, "Random matrix theory and wireless communications," Foundations and Trends in Commun. and Info. Theory, vol. 1, no. 1, pp. 1-182, 2004.

[12] R. Couillet and M. Debbah, Random Matrix Methods for Wireless Communications, 1st ed. Cambridge University Press, 2011.

[13] M. Matthaiou, M. Mckay, P. Smith, and J. Nossek, "On the condition number distribution of complex wishart matrices," IEEE Trans. Commun., vol. 58, no. 6, pp. $1705-1717$, June 2010.

[14] Y. Zeng and Y.-C. Liang, "Spectrum-sensing algorithms for cognitive radio based on statistical covariances," IEEE Trans. Veh. Technol., vol. 58, no. 4, pp. $1804-1815$, May 2009.

[15] M. Naraghi-Pour and T. Ikuma, "Autocorrelation-based spectrum sensing for cognitive radios," IEEE Trans. Veh. Technol., vol. 59, no. 2, pp. 718 -733 , Feb. 2010.

[16] L. Cardoso, M. Debbah, P. Bianchi, and J. Najim, "Cooperative spectrum sensing using random matrix theory," in 3rd Int. Symp. Wireless Pervasive Computing, May 2008, pp. $334-338$. 
[17] C. Tepedelenlioglu and R. Challagulla, "Low-complexity multipath diversity through fractional sampling in OFDM," IEEE Trans. Signal Process., vol. 52, no. 11, pp. $3104-3116$, Nov. 2004.

[18] Y. Imaoka and Y. Sanada, "Experimental investigation of sampling rate selection with fractional sampling for IEEE802.11b WLAN system," in IEEE 70th Veh. Technol. Conf., Sept. 2009, pp. 1 -5.

[19] H. Nishimura, M. Inamori, and Y. Sanada, "Sampling rate selection for fractional sampling in OFDM," in IEEE 18th Int. Symp. Personal, Indoor and Mobile Radio Commun. (PIMRC), Sept. 2007, pp. 1 -5.

[20] B. Li, Q. Wang, G. Lu, Y. Chang, and D. Yang, "Linear MMSE frequency domain equalization with colored noise," in IEEE 66th Veh. Technol. Conf., Sept. 30-oct. 3 2007, pp. $1152-1156$.

[21] F. Penna, R. Garello, D. Figlioli, and M. Spirito, "Exact non-asymptotic threshold for eigenvalue-based spectrum sensing," in 4th Int. Conf. Cognitive Radio Oriented Wireless Networks and Commun. (CROWNCOM), June 2009, pp. $1-5$.

[22] F. Penna, R. Garello, and M. Spirito, "Cooperative spectrum sensing based on the limiting eigenvalue ratio distribution in Wishart matrices," IEEE Commun. Lett., vol. 13, no. 7, pp. 507 -509, July 2009.

[23] G. de Abreu, W. Zhang, and Y. Sanada, "Spectrum sensing algorithms via finite random matrix theory," in IEEE Int. Conf. Commun. (ICC), June 2011, pp. $1-5$.

[24] L. Wang, B. Zheng, J. Cui, and W. Yue, "Spectrum sensing using nonasymptotic behavior of eigenvalues," in Int. Conf. Wireless Commun. and Signal Process., Nov. 2011, pp. 1 -5.

[25] K. Cao and Z. Yang, "A novel cooperative spectrum sensing algorithm based on random matrix theory," in 6th Int. Conf. Wireless Commun. Networking and Mobile Comput., Sept. 2010, pp. $1-4$.

[26] K. Hassan, R. Gautier, I. Dayoub, E. Radoi, and M. Berbineau, "Predicted eigenvalue threshold based spectrum sensing with correlated multiple-antennas," in IEEE 75th Veh. Technol. Conf. (VTC Spring), May 2012, pp. $1-5$.

[27] L. Wang, B. Zheng, J. Cui, S. Tang, and H. Dou, "Cooperative spectrum sensing using free probability theory," in IEEE Global Telecommun. Conf., Dec. 2009, pp. 1 -5.

[28] L. Wang, B. Zheng, J. Cui, and Q. Meng, "Cooperative MIMO spectrum sensing using free probability theory," in 5th Int. Conf. Wireless Commun., Networking and Mobile Comput., Sept. 2009, pp. 1 -4.

[29] M. Grimm, R. K. Sharma, M. Hein, and R. Thoma, "Non-linearly induced interference and its mitigation in cognitive wideband receivers," 18th European Wireless Conf., pp. 1 -6, April 2012.

[30] A. Vasilopoulos, G. Vitzilaios, G. Theodoratos, and Y. Papananos, "A low-power wideband reconfigurable integrated active RC filter with 73 dB SFDR," IEEE J. Solid-State Circuits, vol. 41, no. 9, pp. 1997 -2008, Sept. 2006.

[31] H. Shin and Y. Kim, "A CMOS active RC low-pass filter with simultaneously tunable high and low cutoff frequencies for IEEE 802.22 applications," IEEE Trans. Circuits and Systems II: Express Briefs, vol. 57, no. 2, pp. $85-89$, Feb. 2010.

[32] S. Haykin, Communication Systems, 4th ed. John Wiley \& Sons, Inc.

[33] Y. Zeng, Y.-C. Liang, A. Hoang, and R. Zhang, "A review on spectrum sensing for cognitive radio: Challenges and solutions," EURASIP J. Advances in Signal Process., vol. 2010, no. 1, pp. 1-15, 2010.

[34] O. Tirkkonen and L. Wei, Foundation of Cognitive Radio Systems: Exact and asymptotic analysis of largest eigenvalue based spectrum sensing. InTech, 2012, no. 978-953-51-0268-7, ch. 1 .

[35] X. Mestre, J. Fonollosa, and A. Pages-Zamora, "Capacity of MIMO channels: asymptotic evaluation under correlated fading," IEEE J. Sel. Areas Commun., vol. 21, no. 5, pp. 829 - 838, June 2003.

[36] S. Loyka, "Channel capacity of MIMO architecture using the exponential correlation matrix," IEEE Commun. Lett., vol. 5, no. 9, pp. 369 -371, Sept. 2001

[37] S. Chatzinotas, M. Imran, and R. Hoshyar, "On the multicell processing capacity of the cellular MIMO uplink channel in correlated Rayleigh fading environment," IEEE Trans. Wireless Commun., vol. 8, no. 7, pp. $3704-3715$, July 2009.

[38] D. Voiculescu, "Multiplication of certain non-commuting random variables," J. Operator Theory, vol. 18, pp. 223-235, 1987.

[39] B. Nosrat-Makouei, J. Andrews, and R. Heath, "MIMO interference alignment over correlated channels with imperfect CSI," IEEE Trans. Signal Process., vol. 59, no. 6, pp. 2783 -2794, June 2011.

[40] S. M. Kay, Fundamentals of Statistical Signal Processing: Detection Theory. Prentice Hall, 1998, vol. II.

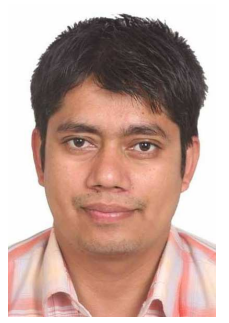

Shree Krishna Sharma (B.E.,M.A.,M.Sc.,M. Res.) received his B. E. in electronics and communication from Birla Institute of Technology (BIT), Mesra, India in 2004 and M. Sc. degree in Information and Communication Engineering from Institute of Engineering (IOE), Pulchowk, Nepal in 2010. He was also awarded an M.A. degree in Economics from Patan Multiple Campus, Tribhuvan University (TU), Nepal and an M. Res. degree in Computing Science from Staffordshire University, UK in 2011. He is currently working towards his Ph.D. degree in the Interdisciplinary Centre for Security, Reliability and Trust, University of Luxembourg under AFR Ph.D. grant from National Research Fund, Luxembourg. He was recipient of the Indian Embassy Scholarship for his B.E. study and the Erasmus Mundus Scholarship for his M. Res. study. He has worked in Nepal Telecom (NT) company as a Telecom Engineer for more than four years in the field of information technology and telecommunication. In the past, he was also involved with Kathmandu University (KU), Nepal as a teaching assistant and he served as a part time lecturer in 8 engineering colleges in Nepal. His research interests include cognitive wireless communications, resource allocation and interference mitigation in heterogeneous wireless networks.

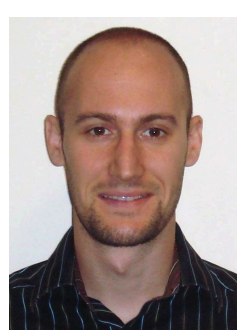

Symeon Chatzinotas (S'06-M'09) received the M.Eng. in Telecommunications from Aristotle University of Thessaloniki, Greece and the M.Sc. and $\mathrm{Ph} . \mathrm{D}$. in Electronic Engineering from University of Surrey, UK in 2003, 2006 and 2009 respectively. $\mathrm{He}$ is currently a Research Scientist in the Interdisciplinary Centre for Security, Reliability and Trust, University of Luxembourg.

In the past, he has worked in numerous $R \& D$ projects for the Institute of Informatics \& Telecommunications, National Center for Scientific Research "Demokritos", the Institute of Telematics and Informatics, Center of Research and Technology Hellas and Mobile Communications Research Group, Center of Communication Systems Research, University of Surrey. He has authored more than 60 technical papers in refereed international journals, conferences and scientific books. His research interests are on multiuser information theory, cooperative and cognitive communications and transceiver optimization for terrestrial and satellite networks.

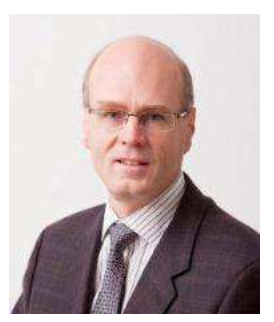

Björn Ottesten (S'87-M'89-SM'99-F'04) was born in Stockholm, Sweden, in 1961. He received the M.S. degree in electrical engineering and applied physics from Linköping University, Linköping, Sweden, in 1986 and the Ph.D. degree in electrical engineering from Stanford University, Stanford, CA, in 1989.

He has held research positions at the Department of Electrical Engineering, Linköping University; the Information Systems Laboratory, Stanford University, Stanford, CA; and the Katholieke Universiteit Leuven, Leuven, Belgium. In 1991, he was appointed Professor of signal processing at the Royal Institute of Technology (KTH), Stockholm, where he was head of the Department for Signals, Sensors, and Systems from 1992 to 2004 and Dean of the School of Electrical Engineering from 2004 to 2008. Since 2009, he has been Director of securityandtrust.lu at the University of Luxembourg. During 1996-1997, he was Director of Research at ArrayComm Inc, San Jose, CA, a start-up company based on Otterstens patented technology. His research interests include wireless communications, stochastic signal processing, sensor array processing, and time-series analysis. Dr. Ottersten has coauthored papers that received an IEEE Signal Processing Society Best Paper Award in 1993, 2001, and 2006, and three IEEE conference papers that received Best Paper Awards. He has served as Associate Editor for the IEEE TRANSACTIONS ON SIGNAL PRECESSING and on the Editorial Board of the IEEE Signal Processing Magazine. He is currently Editorin-Chief of the EURASIP Signal Processing Journal and a member of the Editorial Board of the EURASIP Journal of Advances Signal Processing. In 2011 he received the IEEE Signal Processing Society Technical Achievement Award. He is a first recipient of the European Research Council advanced research grant. 